\title{
A Secure Distributed Video Surveillance System Based on Portable Devices
}

\author{
Pietro Albano ${ }^{1}$, Andrea Bruno ${ }^{1}$, Bruno Carpentieri ${ }^{1}$, Aniello Castiglione ${ }^{1}$, \\ Arcangelo Castiglione ${ }^{1}$, Francesco Palmieri ${ }^{2}$, \\ Raffaele Pizzolante ${ }^{1, \star}$, and Ilsun $\mathrm{You}^{3}$ \\ 1 Dipartimento di Informatica "R.M. Capocelli" \\ Università degli Studi di Salerno \\ I-84084, Fisciano (SA), Italy

\begin{abstract}
In this work a distributed video surveillance system based on a Client-Server architecture is presented. The proposed system is accessible from portable devices such as tablets, smartphones, etc. In a typical real-world scenario, for example in homeland security, it is useful to have portable devices that can receive in real-time a frame or a sequence of frames coming from a selected camera to prevent or to detect attacks (i.e. terrorist attacks, etc.). In the proposed system, a portable device knows only the address of the server (repository), and the repository sends to the portable device the list of the clients (nodes) which are connected with one or more cameras. When the portable device obtains the list of the nodes, it connects directly to a specific node and requests the images of its connected cameras. The whole system provides secure communication channel between all its components. The security of both the node-repository and the repository-portable devices communications is guaranteed by using a secure connection. The security of the node-portable devices interconnection is provided by a digital invisible watermarking algorithm that affects each image before sending it from the node to the portable devices. Each portable device can extract the watermark and verify the identity of the node.
\end{abstract}

Keywords: Video Surveillance, Remote Video Surveillance, Remote Personal Security, Mobile Video Surveillance, Remote Homeland Security.

^ Corresponding author.

G. Quirchmayr et al. (Eds.): CD-ARES 2012, LNCS 7465, pp. 403-415, 2012.

(C) IFIP International Federation for Information Processing 2012 


\section{Introduction}

Video surveillance has become increasingly important in everyday use and video surveillance systems have become sophisticated and are increasingly accessible and usable by the general public. Important applications of video surveillance are the identification of individuals and objects as well as the prevention and the detection of abnormal activities. Video surveillance is also helpful in other fields such as agriculture (for the prevention of fires), etc.

With the diffusion of advanced portable devices, now it is possible to perform surveillance and monitoring activities using them.

Hence, in this work we propose a secure distributed system for video surveillance, based on a Client-Server paradigm, that introduces the possibility of remote connections from portable devices for real-time monitoring. The system architecture is based on several basic entities: a central server (or repository), which knows the locations of some collector nodes, connected with one or more cameras.

The portable devices accessing the system know only the location of the repository. When the connection is established, the repository sends to the portable device a list of nodes and, as a second step, the device connects directly with a node and receives from it the image frames or multimedia contents obtained by its camera(s). There is an high degree of security both in the communication among the system parts and in the video frames.

The remainder of this work is organized as follows: Section 2 discusses the distributed architecture of the proposed system. Section 3 focuses on the security aspects and describes the approaches for the engineering and development of the prototype we have developed. In Section 4 we describe the system from the point of view of the end user and in Section [5] we present our conclusions and highlight future work directions.

\section{The Distributed Architecture}

The main task of the repository is to allow the localization of the nodes. It interacts with them and with the portable devices accessing the system by maintaining a list of all the nodes that joined the system.

A collector node is connected with at least a camera and interacts with the portable devices interested in monitoring the areas covered by its associated camera(s). The portable devices need to know only the IP address of the repository that provides the IP addresses of the list of the available collector nodes and other descriptive information about them. When a portable device connects to a node it receives, at regular intervals, the images obtained by the specific camera(s) connected to the node.

Figure 1 shows a graphical representation of the proposed system architecture.

In the following, we discuss the interaction among node-repository (see Section 2.1), repository-portable device (see Section 2.2) and node-portable device (see Section 2.3). 


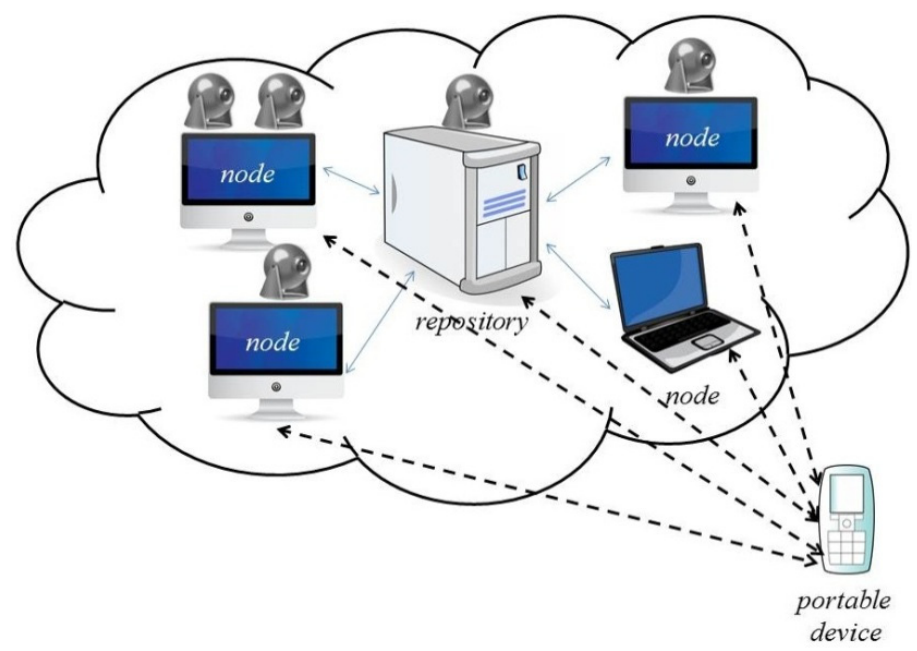

Fig. 1. The architecture of the proposed system

\subsection{Interaction Node-Repository}

The communication between these two entities takes place through a TCP connection over TLS/SSL. Its security is essential, since without a secure connection a fake node could easily enter into the system and send manipulated/tampered images to the accessing portable devices.

There are three types of messages exchanged between a node and the repository: Login, Register, and Disconnect. These messages are encoded by XML markup sequences. Figure 2 shows an example of a complete interaction between the above entities, including each of the three messages.

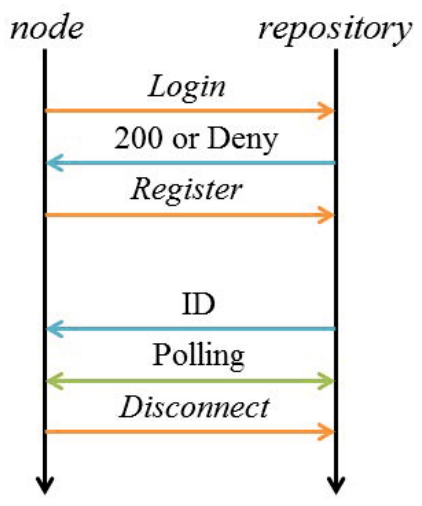

Fig. 2. An example of a complete interaction between a node and the repository 
The Login message is used by a node to authenticate itself to the repository. If the authentication succeeds, the repository answers with a return code "200", otherwise the repository denies the access for that node. Figure 3 shows an example of Login message from a node to the repository.

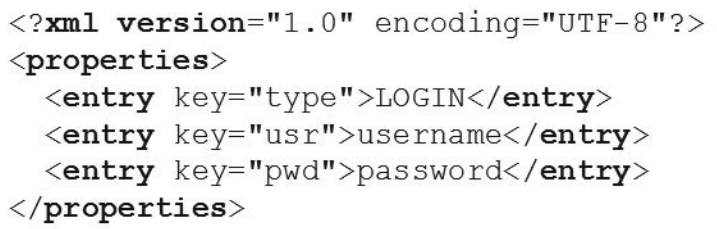

Fig. 3. An example of a Login message from a node to the repository

The Register message is used by a node to send its descriptive information to the repository. When the repository receives the information about the node it replies with an $I D$. The $I D$ is also used by the node during the disconnection process. Figure 4 shows an example of Register message from a node to the repository.

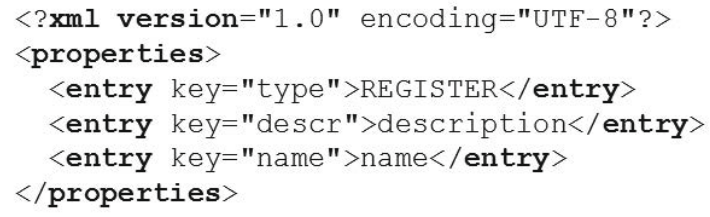

Fig. 4. An example of a Register message from a node to the repository

When a node want to disconnect itself from the repository, it sends Disconnect message as shown in Figure 5.

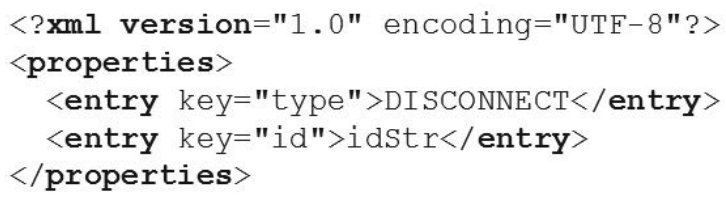

Fig. 5. An example of a Disconnect message from a node to the repository

\subsection{Interaction Repository-Portable Device}

As previously, the interaction between the repository and the portable devices is based on a TCP connection over TLS/SSL. There are three new commands associated respectively to three different XML messages: ListServer, Update and 
Desc. The ListServer message requests the server to download the list of nodes currently registered. The reply of the repository can be either the " 530 " return code in case of error, or a string having this form:

\section{$/ / n \% t_{s} \% \mathrm{ID}_{1} \% \mathrm{Name}_{1} \% \mathrm{IPAddress}_{1} \% \% \ldots \% \mathrm{ID}_{n} \%$ Name $_{n} \%$ IPAddress $_{n} \% \% / /$}

where:

$\boldsymbol{n}$ is the number of the entries;

$\boldsymbol{t} \boldsymbol{s}$ is a timestamp which is used for indicate when the request has been sent and is also used with Update command;

ID $_{i}$ is an unique identifier associated to node $i$

Name $_{\boldsymbol{i}}$ is the alias or the name of the node $i$;

IPAddress $_{i}$ is the IP address of the node $i$.

The Update message is used by the node to communicate to the repository its intention to update the local list. The command Update takes as parameter the timestamp, used by the repository to verify if the list of nodes maintained by the portable device has been updated. The possible replies of the repository can be: a "400" return code if there isn't updates or, alternatively, a string structured as:

$$
\begin{gathered}
/ / n \% t_{s} \% \%\left[+\mid-\mathrm{ID}_{1} \% \text { Name }_{1} \% \text { IPAddress }{ }_{1} \% \% \ldots\right. \\
\% \%[+\mid-] \mathrm{ID}_{n} \% \text { Name }_{n} \% \text { IPAddress } n \% / /
\end{gathered}
$$

where:

$\boldsymbol{n}$ is the number of the entries $(n)$;

$\boldsymbol{t} \boldsymbol{s}$ is a timestamp which is used to indicate when the request has been sent;

$+\mathbf{I D}_{\boldsymbol{i}}$ is the node with the identifier ID $i$ has been registered after the last request of update, or

$-\mathbf{I D}_{i}$ is the node with the identifier ID $i$ has been disconnected after the last request of update;

Name $_{\boldsymbol{i}}$ is the alias or the name of the node $i$;

IPAddress $_{i}$ is the IP address of the node $i$.

\subsection{Interaction Node-Portable Device}

The communication between these two entities is performed through the use of TCP sockets which adopt a FTP-like protocol for exchanging messages and data. The main difference between the FTP protocol and the proposed ad-hoc protocol is that in the former the client opens two communication channels (one for the messages and one for the data), in the latter, portable device, using a GPRS/UMTS connection, cannot open more than one channel due to commonly closed mobile operators policies.

In the proposed protocol the server (node) opens two sessions (with two different channels) and the client (the portable device) opens just one. The node communicates to the client the port of the other opened channel, resulting in two communication options. The three message used are: Login, List, and MGet. 
The Login message is used by the portable device to authenticate. The Login operation can succeed or not. In the first case the node replies to the portable device with a message with return code " 200 " and the communication continues normally. On the contrary, the portable device receives an alert message on the display.

The List message is used by the portable device to request a snapshot of all the environments monitored by the node. When a node receives this message, it takes a snapshot from each of its camera(s) and sends it to the portable device.

The MGet message is used by the portable device to request the monitoring of a specific area, identified by an unique identifier. When this message is received by the node, it opens a data channel that is able to send the multimedia frames at regular intervals.

\section{System Security}

The development of secure architectures, providing controlled access, privacy protection, content confidentiality and authenticity, is one of the most challenging issues in the video surveillance area, and several solutions, based on the use of cryptography have been proposed (e.g. [1, 2, 3]). Furthermore, the existence of a surveillance system strongly depends on legal boundaries 4 that states what is allowed to be monitored, what is not, and also who is authorized to perform monitoring. In these cases the data produced by surveillance activities must be properly secured against unauthorized accesses or misuses of the collected images.

The proposed system has two important security aspects: the first one concerns the communication channel between a node and the repository, while the second one concerns the communication channel between a node and a portable device.

In the first case the secure connection between the repository and a node is guaranteed by using a SSL/TLS connection, providing privacy through symmetric cryptography and message reliability through keyed message authentication codes. Asymmetric cryptography is also used to protect each key exchange. This ensures the prevention against any type of eavesdropping and tampering.

However, some portable devices could not be able to implement, fully or partially, the aforementioned cryptographic mechanisms and primitives. For this reason we decided to use Digital Watermarking techniques to further guarantee security between a node and a portable device.

Therefore, each node, before sending an image modifies it with an invisible digital watermark. On the other hand, when the portable device receives the image, it extracts the watermark in order to verify the authenticity of each image.

\subsection{Digital Watermarking to Improve the Security of the Proposed System}

Digital Watermarking is one of the techniques generally used to insert hidden data into digital contents. When a signal is protected by a robust digital watermark, then the associated hidden information will be also carried in its copies. Watermarking is also used to prevent unauthorized copy of digital media. 
There are different embedding methods: such as Spread-Spectrum [5, 6, 7, or Amplitude Modulation [8].

In the first one, the signal affected by digital watermark is obtained by an additive modification. Also in the case of amplitude modulation the marked signal is obtained by an additive modification, like in Spread-Spectrum embedding, but the watermark is only embedded in the spatial domain.

Before sending the images, the node embeds in them a digital invisible watermark. When the portable device receives the image it extracts the watermark in order to verify the trueness of each image.

The watermarking algorithm used in this work has been proposed in [9], and it is based on a modified version of the one proposed in Langelaar et al. [10].

It takes as input the source image, the watermark string, a seed and a threshold $T$.

The watermark string is converted in a bit matrix where each character is converted in a 5x8 sub-matrix of bits (an example is reported in Figure 6). The resulting bit string is obtained by reading the bit matrix line-by-line from left-top corner. The seed represents an ID (such as numeric PIN) that is used to embed the watermark, and in second instance to extract it from the watermarked image. The threshold $T$ is a real number that indicates the robustness of the watermark that will be embedded.

The algorithm used for embedding a digital invisible watermark in an image is sketched as follows:

1. The image is converted from the RGB domain to the YUV domain.

2. The watermark string is converted to a matrix of bits. Each character is converted in a $5 \times 8$ matrix of bits (see the example in Figure 6).

The resulting matrix will be embedded in the original image line-by-line from left-top corner.

3. A block $B$ of $8 \times 8$ pixels is pseudo-randomly selected from the image to embed one bit of the watermark string.

4. A fixed binary pseudo-random pattern of the same size of $B$ is generated.

5. The $I_{0}, I_{1}$ and $D$ quantities are calculated from $B . I_{0}$ and $I_{1}$ are obtained by calculating the averages of the luminance values in $B$, respectively where the random pattern is 0 and where the random pattern is $1 . D$ is the difference $I_{1}-I_{0}$.

6. $B^{\prime}$ is a reduced quality block obtained by applying the quantization and the 8x8 DCT transform.

7. The $I_{0}^{\prime}, I^{\prime}{ }_{1}$ and $D^{\prime}$ quantities are calculated from $B^{\prime} . I^{\prime}{ }_{0}$ and $I^{\prime}{ }_{1}$ are obtained by calculating the averages of the luminance values in $B^{\prime}$, respectively where the random pattern is 0 and where the random pattern is $1 . D^{\prime}$ is the difference $I^{\prime}{ }_{1}-I^{\prime}{ }_{0}$.

8. If the bit to embed has value 1 the go to step 11 .

9. In order to embed the bit with value 0 , the binary pseudo-random pattern is subtracted from the block $B$, if $D$ and $D^{\prime}$ are greater than the threshold $T$. The steps $6-8$, and 10 are repeated iteratively until both differences are less or equal than $-T$. Go to step 12 . 
10. In order to embed the bit with value 1 , the binary pseudo-random pattern is added from the block $B$, if $D$ and $D^{\prime}$ are less or equal than the threshold $T$. The steps $6-8$, and 11 are repeated iteratively until both differences are greater than $T$.

11. The steps from 4 to 11 are applied to all pseudo-randomly selected blocks until all bits of the watermark string are embedded.

12. The image in YUV domain is converted back to the RGB domain.

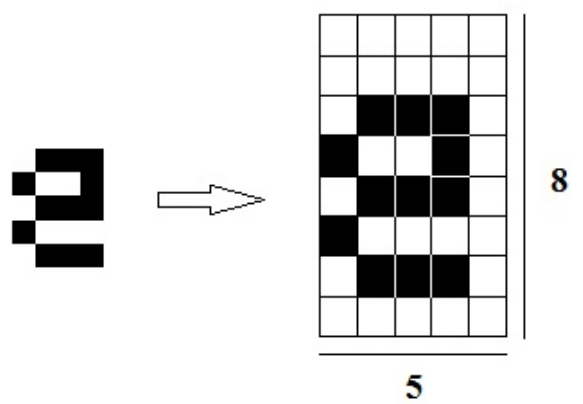

Fig. 6. Example of conversion from the character 'e' to the matrix of bits composed by $5 \times 8$ (40 bits). The white cells are represented by the value 0 and the black cells are represented by the value 1 .

Figure 7(a) and Figure 7(b) show respectively the original "Lena" image and the "Lena" image affected by a digital invisible watermark of 200 bits (the string "SeCAM") with the previously described watermarking algorithm.

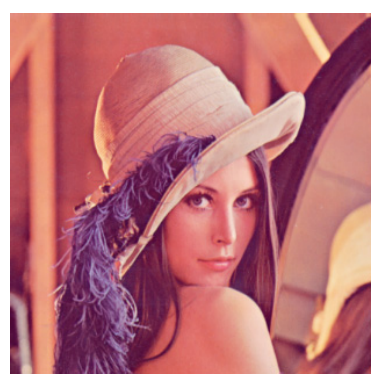

(a)

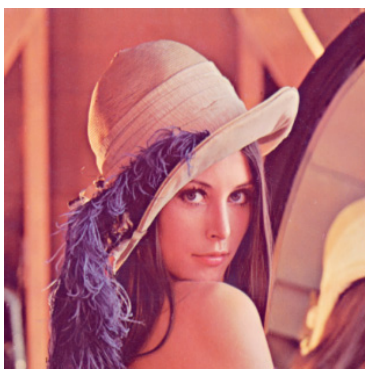

(b)

Fig. 7. (a) The original "Lena" image; (b) The "Lena" image affected by a digital invisible watermark

Analogously, the algorithm for extracting the digital invisible watermark is reported below: 
1. The image is converted from the RGB domain to the YUV domain.

2. A block $B$ of $8 \times 8$ pixels is pseudo-randomly selected from the image to read one bit of the watermark string.

3. A fixed binary pseudo-random pattern of the same size of $B$ is generated.

4. $I_{0}, I_{1}$ and $D$ are calculated from $B . I_{0}$ and $I_{1}$ are then obtained by calculating the averages of the luminance values in $B$, respectively where the random sequence is 0 and where the random sequence is 1 . $D$ is the difference $I_{1}-I_{0}$.

5. If $D>0$ then the embedded bit has value 1 else the embedded bit has value 0 .

\section{The End User Interface}

From the end user point of view, there are three main system components:

- The repository GUI (described in Section 4.1)

- The node GUI (described in Section 4.2)

- The portable device GUI (described in Section 4.3)

\subsection{The Repository GUI}

The repository GUI is very intuitive and simple, it is subdivided in two parts: the first one provides a panel to configure its starting options (Username and Password), the second one provides a panel which allows the repository to operate as a node. An example is shown in Figure 8.

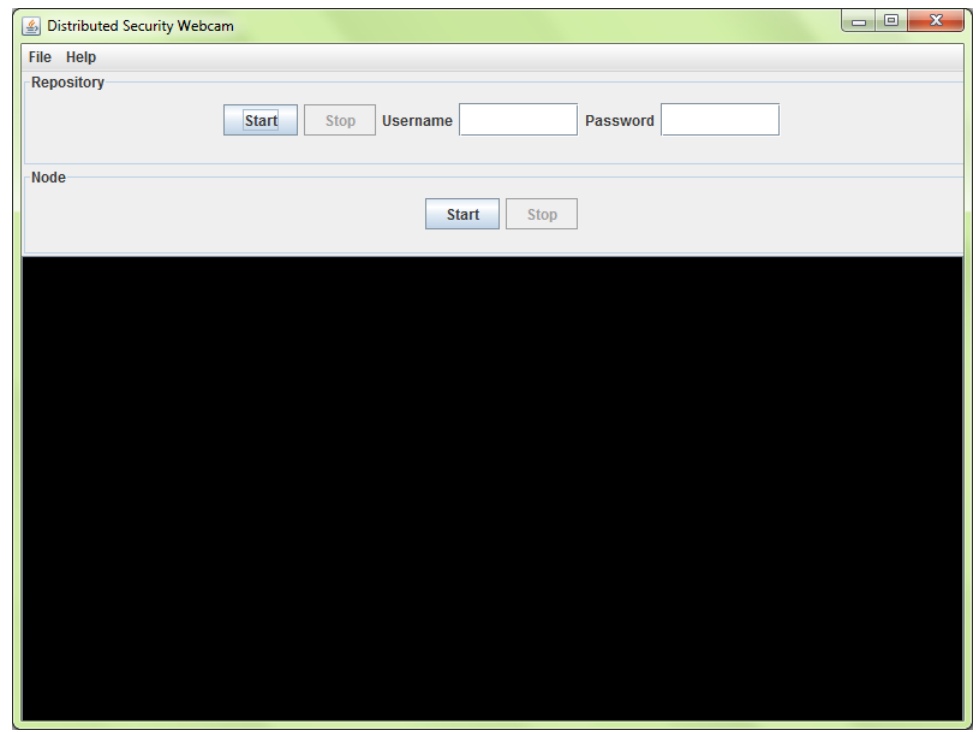

Fig. 8. The repository GUI 


\subsection{The Node GUI}

The node GUI takes as input from the user: the Username and the Password, obtained during the registration process, the Name (or the alias) of the node, the Hostname (that is the IP address of the repository) and the Description of the node. Figure 9 shows an example of the node GUI.

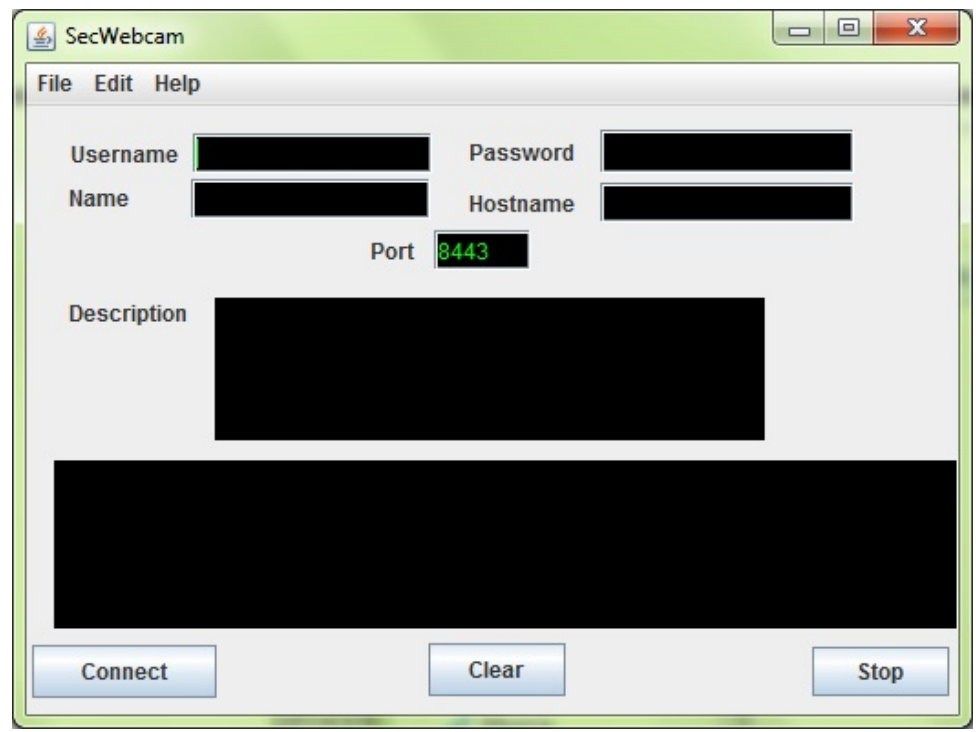

Fig. 9. The node GUI

\subsection{The Portable Device GUI}

As in the case of the repository GUI, also the one of portable device is subdivided in two parts. In the first step (Figure 10(a) the end user must insert the information associated to the connection with the repository. In details, the required information are: Username, Password and Host respectively used for authentication and connection.

In the second step (Figure 10(b) the application shows the list of nodes obtained from the repository. For each node, the application has three commands: Details, Update and Connect. The Details command permits to obtain a detailed description of the node. The Update command permits to request the update of the local list of the nodes. The Connect command permits to connect with the node and to obtain the sequences of frames captured by the camera(s) (Figure 10(c) of the node. Moreover, the Exit command permits to disconnect and close the application. 


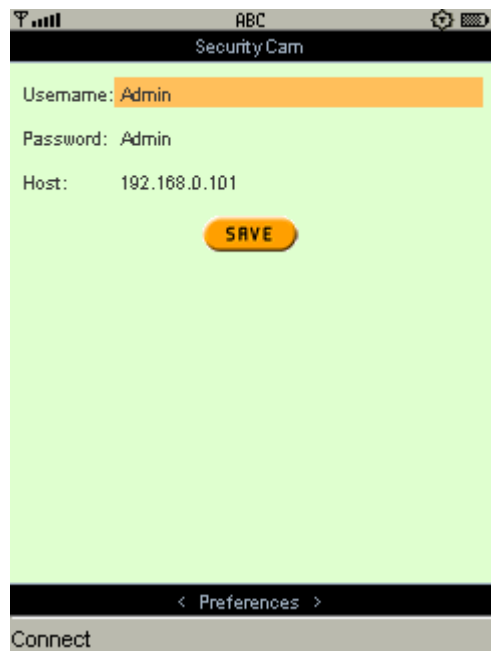

(a)
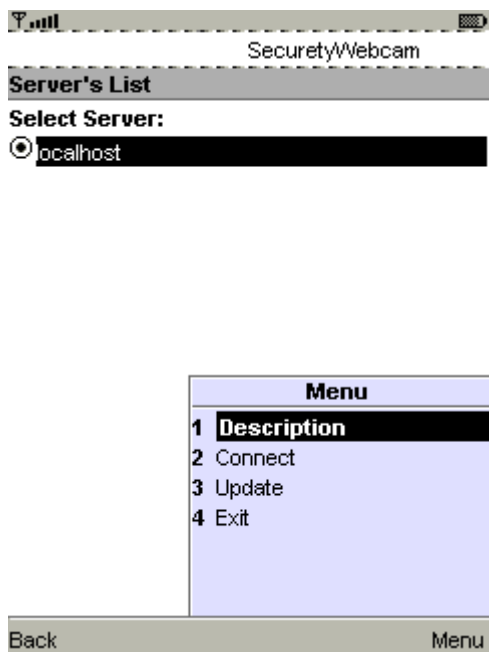

(b)

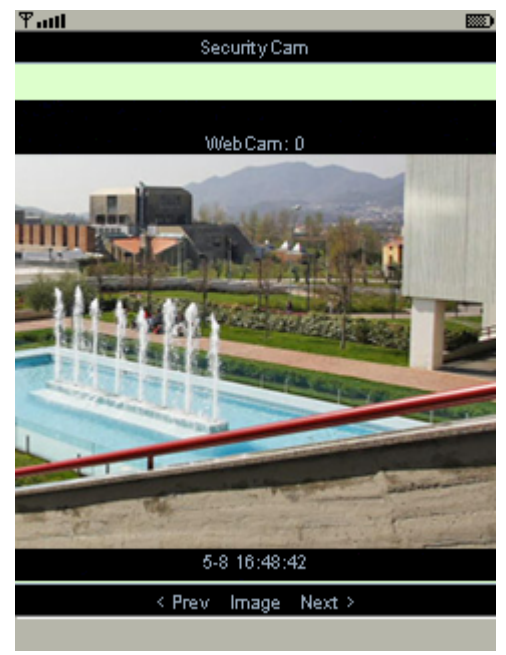

(c)

Fig. 10. (a)shows an example of the first step of the portable device GUI; (b) shows an example of the portable device GUI with the list of the nodes; (c) shows an example of the image obtained by the selected camera of the node 


\section{Conclusion and Future Works}

In recent years, video surveillance has become an important system for the monitoring of areas, environments, etc. One of the more important application of the video surveillance is the security, but video surveillance is used also for other purposes such as traffic monitoring, etc.

In a real-world scenario, as for instance the homeland security and defense issues, where the video surveillance systems assumes a critical role, it is useful to receive real-time images from different camera(s), directly on a portable device as allowed by the presented system in this paper.

With the wide diffusion of portable devices such as tablets, smartphones and so on, it is now meaningful to have a really secure video surveillance system accessible from these devices, to reinforce or to extend the existent video surveillance systems.

The presented distributed video surveillance system, accessible from portable devices, is based on Client-Server architecture. A portable device that joins the system knows the address of the repository and obtains the complete list of the nodes which are connected to one or more camera(s). When the portable device obtains the list, it connects directly to a specific node and obtains the images from the camera(s).

Future work will consider the extension of the proposed architecture to a peerto-peer architecture to allow scalability of the system with an increasing number of nodes.

Another research aspect could be the support for video-streaming from a node to the portable devices. Video streaming could allow advanced commands such as Play, Pause, Stop and FrameCapture respectively to indicate to node that can start the video streaming, that can temporarily stop the streaming, that can stop and deallocate the resources of the video streaming and to indicate to save a frame during the streaming, that, in second time, could send it to the portable device.

Moreover, it could be of interest to consider the sending from a node to a portable device a compressed short video with audio.

\section{References}

[1] Castiglione, A., Cepparulo, M., De Santis, A., Palmieri, F.: Towards a Lawfully Secure and Privacy Preserving Video Surveillance System. In: Buccafurri, F., Semeraro, G. (eds.) EC-Web 2010. LNBIP, vol. 61, pp. 73-84. Springer, Heidelberg (2010)

[2] Castiglione, A., De Santis, A., Palmieri, F.: Ensuring privacy and confidentiality in digital video surveillance systems. In: Yee, G.O.M. (ed.) Privacy Protection Measures and Technologies in Business Organizations: Aspects and Standards, pp. 245-267. IGI Global (2012)

[3] Fleck, S., Straßer, W.: Towards secure and privacy sensitive surveillance. In: Proceedings of the Fourth ACM/IEEE International Conference on Distributed Smart Cameras, ICDSC 2010, pp. 126-132. ACM, New York (2010) 
[4] Hunker, J., Probst, C.W.: Insiders and insider threats - an overview of definitions and mitigation techniques. Journal of Wireless Mobile Networks, Ubiquitous Computing, and Dependable Applications (JoWUA) 2(1), 4-27 (2011)

[5] Liang, Q., Ding, Z.: Spread spectrum watermark for color image based on wavelet tree structure. In: 2008 International Conference on Computer Science and Software Engineering, vol. 3, pp. 692-695 (December 2008)

[6] Wang, Y.P., Chen, M.J., Cheng, P.Y.: Robust image watermark with wavelet transform and spread spectrum techniques. In: Conference Record of the ThirtyFourth Asilomar Conference on Signals, Systems and Computers (October 29November 1), vol. 2, pp. 1846-1850 (2000)

[7] Bender, W., Gruhl, D., Morimoto, N., Lu, A.: Techniques for data hiding. IBM Systems Journal 35(3.4), 313-336 (1996)

[8] Kutter, M., Jordan, F.D., Bossen, F.: Digital watermarking of color images using amplitude modulation. Journal of Electronic Imaging 7, 326-332 (1998)

[9] Pizzolante, R., Carpentieri, B.: Copyright protection for images on mobile devices. In: Proceedings of IMIS 2012 (2012)

[10] Langelaar, G.C., van der Lubbe, J., Biemond, J.: Copy protection for multimedia data based on labeling techniques. In: 17th Symposium on Information Theory in the Benelux (1996) 